\title{
Theory Of Fractionally Spaced Cyclic-Prefix Equalizers
}

\author{
P. P. Vaidyanathan and Bojan Vrcelj \\ Dept. Electrical Engineering, California Institute of Technology \\ Pasadena, CA 91125, USA \\ ppvnath@systems.caltech.edu_bojan@systems.caltech.edu
}

\begin{abstract}
The cyclic prefix system is widely used for frequency domain equalization in discrete multitone channels. In this paper we show how the idea of fractionally spaced equalization (FSE) can be adapted to cyclic prefix systems. We derive the condition for a perfect FSE, and show that there is a certain freedom in the choice of the equalizer coefficients. This freedom is then exploited to minimize the effect of additive noise at the detector input. The theory is generally applicable to any deconvolution problem, though the setting used for our development uses the language of digital communication.
\end{abstract}

\section{INTRODUCTION}

The cyclic prefix system is widely used for frequency domain equalization in discrete multitone (DMT) channels. In this paper we show how the idea of fractionally spaced equalization (FSE) can be adapted to cyclic prefix systems. In Sec. III we derive the condition for a perfect FSE, and show that there is a certain freedom in the choice of the equalizer coefficients. In Sec. III.3 we show how to take advantage of this freedom to minimize the effect of noise. Examples are presented in Sec. IV, demonstrating the performance of the FSE cyclic prefix system.

The models shown in Fig. 1 are basic to our discussions. In Fig. 1(a) we have a distortion (e.g., channel) with transfer function $C(z)=\sum_{n=0}^{L} c(n) z^{-n}$, additive noise $e(n)$, and a compensator (equalizer in a communications setting) $F(z)$. Here $c(n)$ and $e(n)$ represent uniformly sampled versions of continuous time quantities $c_{c}(t)$ and $e_{c}(t)$, with some sample spacing $T$. A perfect equalizer or zero-forcing equalizer $F(z)=1 / C(z)$ eliminates ISI completely, that is, $\widehat{s}(n)=s(n)$ in absence of noise. The spacing $T$ between the samples $c(n)$ is also the spacing between the symbols $s(n)$, so $F(z)$ is a symbol spaced equalizer (SSE). Figure 1(b) shows the schematic of a fractionally spaced equalizer or FSE [4]. The decimator and expander have standard meanings [6]. Thus $[x(n)]_{\downarrow M}=x(M n)$, and

$$
[x(n)]_{\uparrow M}= \begin{cases}x(n / M) & n \text { a mul. of } M \\ 0 & \text { otherwise. }\end{cases}
$$

Even though we have used the same notations $C(z)$, and $e(n)$ in both figures for simplicity, they are oversampled versions in Fig. 1(b), that is, $c(n)=c_{c}(n T / 2)$ and $e(n)=e_{c}(n T / 2)$. We rarely refer to Fig. 1(a), so there should be no confusion. The symbol stream we wish to

\footnotetext{
${ }^{1}$ Work supported in part by the ONR grant N00014-99-11002, USA.
}

transmit is $s(2 n)$, and its expanded version is $s(n)$. Any interpolation filter at the transmitter is assumed to be absorbed into $C(z)$. The FSE $F(z)$ works at the higher rate $2 / T$. The equalized output $\widehat{s}(n)$ is then decimated. In absence of noise the best thing to do would be to make $\widehat{s}(2 n)=s(2 n)$. This is analogous to the zeroforcing equalizer but the condition is less stringent [4] than $F(z)=1 / C(z)$

(a)

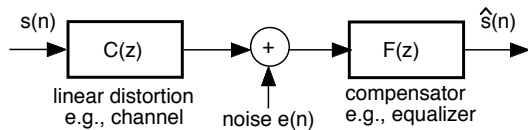

(b)

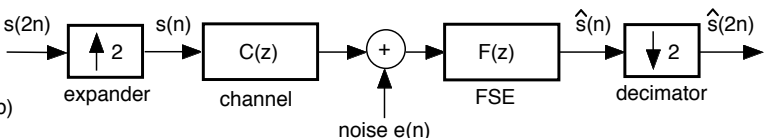

Figure 1. (a) The symbol spaced equalizer (SSE) for a channel $C(z)$, and (b) a fractionally spaced equalizer (FSE).

\section{CYCLIC-PREFIX REVIEW}

Figure 2(a) shows the input stream divided into blocks of length $M$. The $L$ symbols at the end of each block (where $L=$ channel order) are copied into the beginning of that block, to form the cyclic prefix [3] (thin lines in Fig. 2(b)). This evidently assumes $L \leq M$. For a given symbol rate, the cyclic-prefix reduces the spacing between samples (Fig. 2(c)). The factor $\gamma=$ $(M+L) / M$ represents the excess bandwidth required for this. Let $\mathbf{s}(n)$ denote the vector of $M$ input symbols $s(n)$ in the $m$ th block, and let $\mathbf{y}(n)$ be the vector of last $M$ output symbols in the $m$ th block: $\mathbf{s}(m)=$ $\left[\begin{array}{llll}s(m M) & s(m M+1) & \ldots & s(m M+M-1)\end{array}\right]^{T}$ and $\mathbf{y}(m)=\left[\begin{array}{llll}y\left(J_{m}\right) & y\left(J_{m}+1\right) & \ldots & y\left(J_{m}+M-1\right)\end{array}\right]^{T}$ with $J_{m}=m(L+M)+L$. Assuming $L<M$, we can show in absence of noise that $\mathbf{y}(m)=\mathbf{C s}(m)$ where $\mathbf{C}$ is a circulant matrix. For example when $L=2$ and $M=4$,

$$
\mathbf{C}=\left(\begin{array}{cccc}
c(0) & 0 & c(2) & c(1) \\
c(1) & c(0) & 0 & c(2) \\
c(2) & c(1) & c(0) & 0 \\
0 & c(2) & c(1) & c(0)
\end{array}\right)
$$

If $C(z)$ is known, we can perform the equalization by 
inverting $\mathbf{C}$ assuming it is nonsingular. Any circulant can be diagonalized with the DFT matrix [2], that is, $\mathbf{C}=\mathbf{W}^{-1} \boldsymbol{\Lambda}_{c} \mathbf{W}$ where $\mathbf{W}$ is the DFT matrix and

$$
\boldsymbol{\Lambda}_{c}=\operatorname{diag}\{C[0], C[1], \ldots C[M-1]\}
$$

Here $^{2} C[k]=\sum_{n=0}^{M-1} c(n) W^{n k}=M$-point DFT of $c(n)$. Thus the implementation of the communication system with cyclic prefix can be represented as shown in Fig. 3. The box labelled "blocking" is a serial to parallel converter (and "unblocking" converts from parallel to serial). The diagonal elements of $\left[\boldsymbol{\Lambda}_{c}\right]^{-1}$ are $1 / C[k]$, and represent DFTdomain equalizers. Since $\mathbf{y}(m)=\mathbf{C s}(m)$, we can draw a schematic version of Fig. 3 as shown in Fig. 4(a). As $\mathbf{W}^{-1}$ is the inverse of $\boldsymbol{\Lambda}_{c}^{-1} \mathbf{W C}$, we can redraw the system as in Fig. 4(b) resembling discrete multitone systems [1], [5]. If $M$ is a power of two, $\mathbf{W}$ and $\mathbf{W}^{-1}$ can be implemented efficiently with FFT.
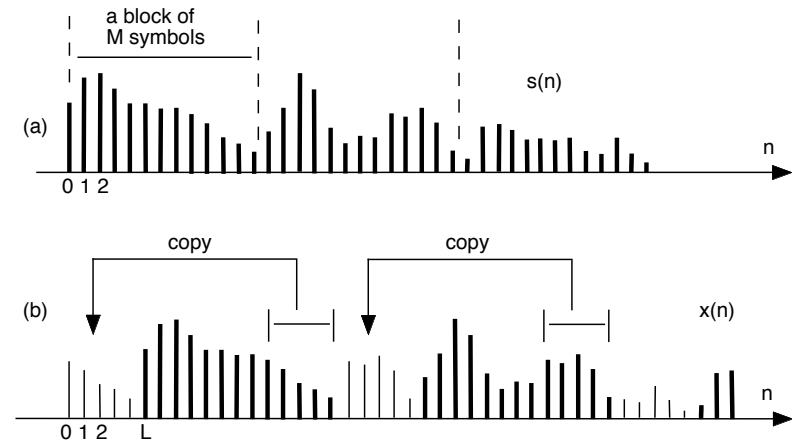

(c)

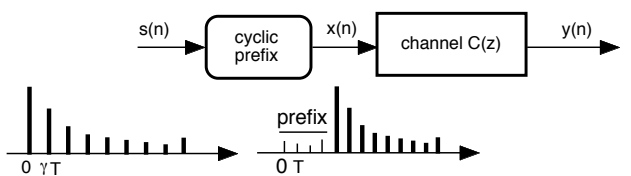

Figure 2. (a) Input symbol stream, (b) cyclic prefix insertion, and (c) block diagram.

(a)
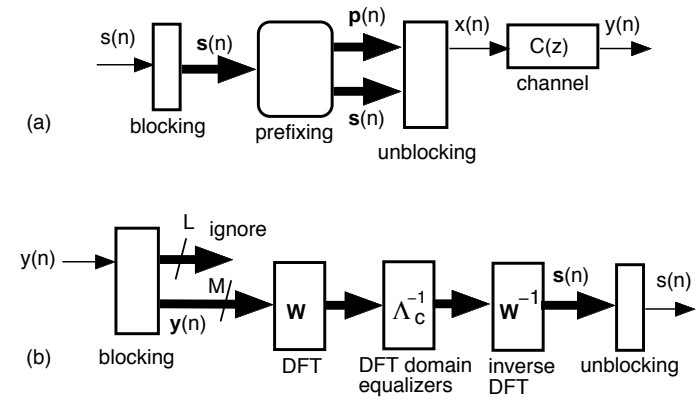

Figure 3. Block diagram description of the cyclic prefix system. (a) Transmitter, and (b) receiver.

\footnotetext{
${ }^{2}$ We use the standard notation $W=e^{-j 2 \pi / M}$.
}
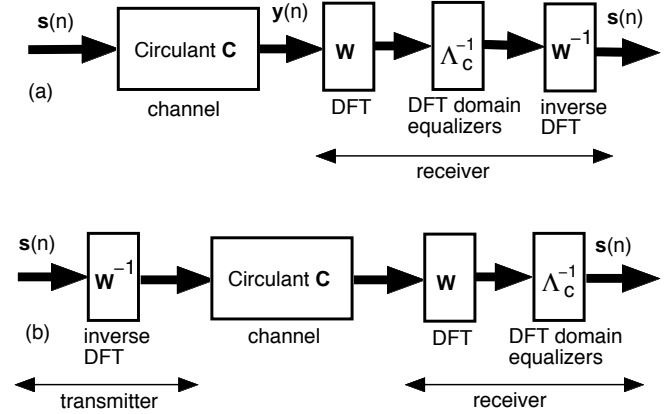

Figure 4. (a) Simplified schematic of the cyclic prefix system, and (b) a useful rearrangement (as in DMT).

\section{THE CYCLIC-PREFIX FSE SYSTEM}

We assume that the portion of Fig. 1(b) from $s(n)$ to $\widehat{s}(n)$ is implemented using the cyclic prefix system. This part can therefore be represented as in Fig. 4(a), and the complete system is as in Fig. 5(a). Here all the matrices are $M \times M$ where $M>L$ with $L$ denoting the order of $C(z)$ (nearly twice the order $L$ in the SSE case). Fig. 5(b) shows the part from the vector $\mathbf{s}(n)$ to the vector $\widehat{\mathbf{s}}(n)$ (use $\left.\mathbf{C}=\mathbf{W}^{-1} \boldsymbol{\Lambda}_{c} \mathbf{W}\right)$. For analysis this figure can further be simplified to Fig. 5(c). The equalizer $\boldsymbol{\Lambda}_{e}$ is diagonal:

$$
\boldsymbol{\Lambda}_{e}=\operatorname{diag}\{E[0], E[1], \ldots E[M-1]\}
$$

\section{III.1. Condition For Perfect Equalization}

Perfect equalization or ISI-free property means $\widehat{s}(2 n)=$ $s(2 n)$ in absence of noise. The obvious choice $\boldsymbol{\Lambda}_{e}=\boldsymbol{\Lambda}_{c}^{-1}$ will achieve $\widehat{\mathbf{s}}(n)=\mathbf{s}(n)$, hence $\widehat{s}(n)=s(n)$. The ISI free property $\widehat{s}(2 n)=s(2 n)$ is less stringent; it only requires that the even numbered components of the vectors $\widehat{\mathbf{s}}(n)$ and $\mathbf{s}(n)$ be identical. The odd components of $\mathbf{s}(n)$ are zero (these are inserted by the expander $\uparrow 2$ in Fig. 1(b)). So only the columns of $\mathbf{W}$ numbered $0,2,4 \ldots$ are active in Fig. $5(\mathrm{c})$. Letting $\mathbf{s}_{1}(n)$ denote the vector obtained by retaining only the even components of $\mathbf{s}(n)$ we can redraw Fig. 5(c) as in Fig. 6(a) for appropriate V.

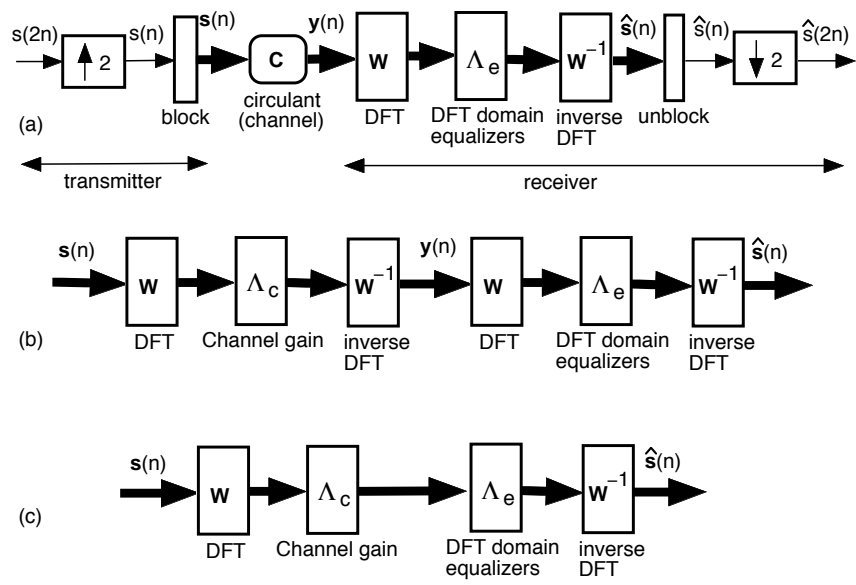

Figure 5. (a) The cyclic prefix system with FSE, and (b), (c) mathematically equivalent forms. 
(a)
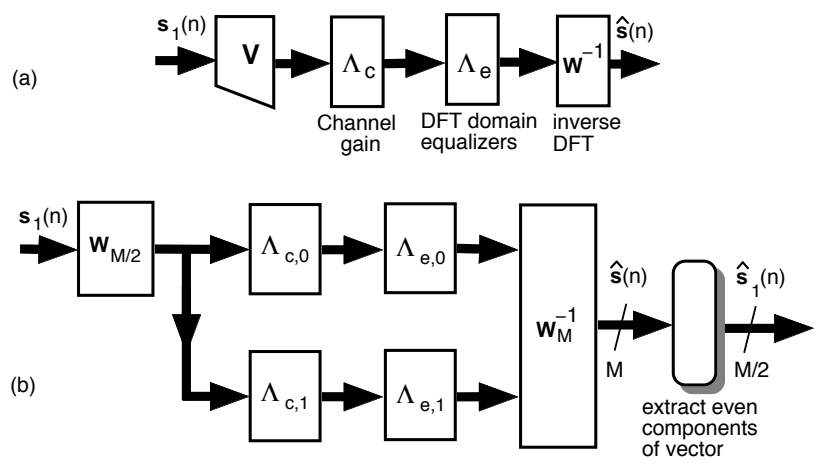

(c)

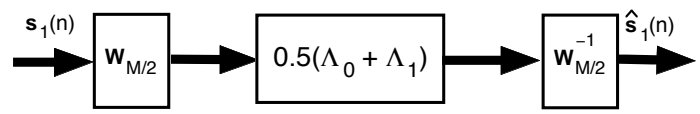

Figure 6. Condition for ISI freedom in cyclic prefix system with FSE. (a), (b), and (c) represent successive stages in the development.

Assume $M$ is even. Since, $W_{M}^{2}=W_{M / 2}$ we have

$$
\mathbf{V}=\left[\begin{array}{l}
\mathbf{W}_{\frac{M}{2}} \\
\mathbf{W}_{\frac{M}{2}}
\end{array}\right]
$$

where $\mathbf{W}_{\frac{M}{2}}$ is the $M / 2$-point DFT matrix. By writing

$$
\boldsymbol{\Lambda}_{c}=\left[\begin{array}{cc}
\boldsymbol{\Lambda}_{c, 0} & \mathbf{0} \\
\mathbf{0} & \boldsymbol{\Lambda}_{c, 1}
\end{array}\right], \quad \boldsymbol{\Lambda}_{e}=\left[\begin{array}{cc}
\boldsymbol{\Lambda}_{e, 0} & \mathbf{0} \\
\mathbf{0} & \boldsymbol{\Lambda}_{e, 1}
\end{array}\right]
$$

we can redraw Fig. 6(a) as in Fig. 6(b). Thus

$$
\widehat{\mathbf{s}}(n)=\mathbf{W}_{M}^{-1}\left[\begin{array}{l}
\boldsymbol{\Lambda}_{0} \\
\boldsymbol{\Lambda}_{1}
\end{array}\right] \mathbf{W}_{\frac{M}{2}} \times \mathbf{s}_{1}(n)
$$

where $\boldsymbol{\Lambda}_{0}=\boldsymbol{\Lambda}_{c, 0} \boldsymbol{\Lambda}_{e, 0}$ and $\boldsymbol{\Lambda}_{1}=\boldsymbol{\Lambda}_{c, 1} \boldsymbol{\Lambda}_{e, 1}$. The final output $\widehat{\mathbf{s}}_{1}(n)$ is obtained by retaining even components:

$$
\widehat{\mathbf{s}}_{1}(n)=\mathbf{W}_{\frac{M}{2}}^{-1}\left[\frac{\boldsymbol{\Lambda}_{0}+\boldsymbol{\Lambda}_{1}}{2}\right] \mathbf{W}_{\frac{M}{2}} \times \mathbf{s}_{1}(n)
$$

This shows that the condition for perfect equalization in the cyclic FSE system is $\boldsymbol{\Lambda}_{0}+\boldsymbol{\Lambda}_{1}=2 \mathbf{I}$, that is,

$$
C[k] E[k]+C[k+M / 2] E[k+M / 2]=2,
$$

for $0 \leq k \leq \frac{M}{2}-1$. Summarizing, we have shown:

Theorem 1. Perfect FSE for cyclic-prefix system. Consider Fig. 1(b) where $C(z)=\sum_{n=0}^{L} c(n) z^{-n}$. Assume that a length- $L$ cyclic prefix is employed at the beginning of each length- $M$ block of $s(n)$ where $M>L$. Then the system is mathematically equivalent to Fig. 5(a). The condition for perfect equalization $(\widehat{s}(2 n)=s(2 n))$ is given by (3) where $C[k]=\sum_{n=0}^{L} c(n) e^{-j 2 \pi k n / M}$ and $E[k]$ are the DFT domain equalizer coefficients (diagonal elements of $\boldsymbol{\Lambda}_{e}$ in Fig. 5(a)).
When $C[k]$ are very small, $1 / C[k]$ tend to amplify channel noise in the SSE system. We do not have this problem for the FSE system because $E[k]=1 / C[k]$ is replaced with (3). In fact for a given $\{C[k], C[k+M / 2]\}$ pair, the equalizer pair $\{E[k], E[k+M / 2]\}$ is not unique. We exploit this in Sec. III.3.

\section{III.2. Structure For The Cyclic-Prefix FSE Receiver}

The receiver for the cyclic-prefix FSE system is reproduced in Fig. 7(a). Notice that this can be redrawn as in Fig. 7 (b). The output of the $M \times M$ IDFT matrix is

$$
\widehat{\mathbf{s}}(n)=\mathbf{W}_{M}^{-1}\left[\begin{array}{cc}
\boldsymbol{\Lambda}_{e, 0} & \mathbf{0} \\
\mathbf{0} & \boldsymbol{\Lambda}_{e, 1}
\end{array}\right] \mathbf{W}_{M} \times \mathbf{y}(n)
$$

The receiver retains only the subvector $\widehat{\mathbf{s}}_{1}(n)$ of even numbered components. This is equivalent to retaining only the rows of $\mathbf{W}_{M}^{-1}$ numbered $0,2,4, \ldots$ Since $W_{M}^{2}=W_{M / 2}$ we can write

$$
\widehat{\mathbf{s}}_{1}(n)=0.5 \mathbf{W}_{M / 2}^{-1}\left[\begin{array}{ll}
\boldsymbol{\Lambda}_{e, 0} & \boldsymbol{\Lambda}_{e, 1}
\end{array}\right] \mathbf{W}_{M} \times \mathbf{y}(n)
$$

The structure for the receiver can therefore be redrawn as in Fig. 7(c). Notice carefully that some vectors have sizes $M$ and some have sizes $M / 2$ as indicated.

(a)
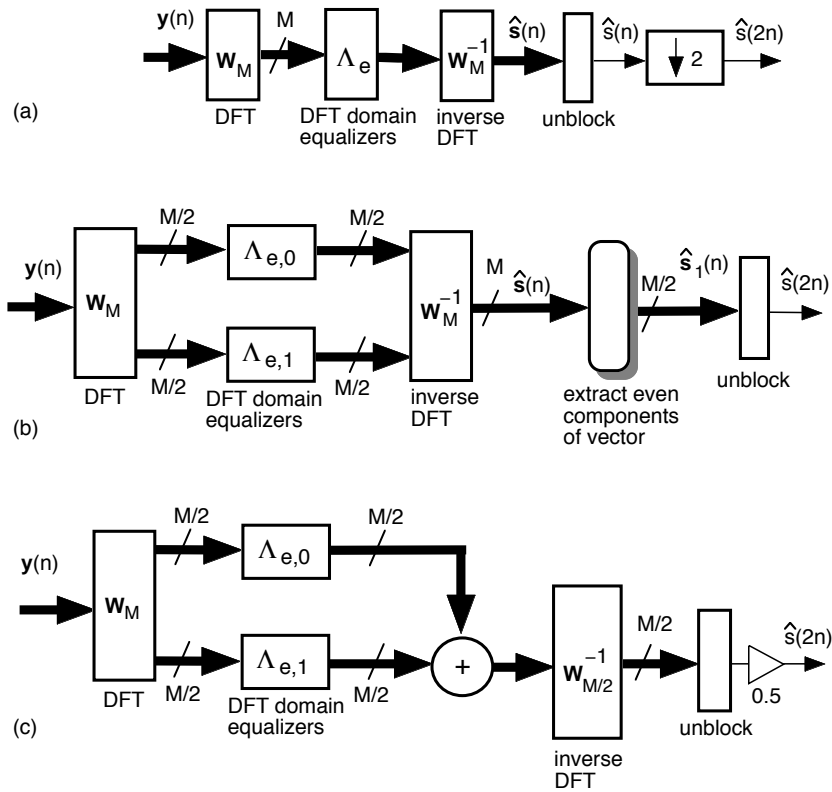

Figure 7. Structure for the FSE receiver in the cyclic prefix system. Parts (a), (b), and (c) represent successive stages in the development.

\section{III.3. Equivalent Structure With IDFT At Transmitter}

We now derive an equivalent structure by moving the IDFT $\mathbf{W}_{M / 2}^{-1}$ in Fig. $7(\mathrm{c})$ to the transmitter side as in conventional DMT systems. Thus consider Fig. 8. Here the matrix $\mathbf{W}_{M / 2}^{-1}$ has been removed from the receiver side and inserted in the transmitter side carefully. We now claim 
that the signal indicated as $\mathbf{t}_{1}(n)$ at the receiver is precisely $\mathbf{s}_{1}(n)$, so that unblocking it yields $s(2 n)$ again (under the obvious assumption than channel noise has been ignored). To see this observe that if $\mathbf{W}_{M / 2}^{-1}$ were inserted again at the receiver as in Fig. $7(\mathrm{c})$, its output would be $\mathbf{r}_{1}(n)$ so that its input would be $\mathbf{W}_{M / 2} \mathbf{r}_{1}(n)=\mathbf{s}_{1}(n)$, as seen from the definitions of signals at the transmitter end in Fig. 8(a). This proves that $\mathbf{t}_{1}(n)=\mathbf{s}_{1}(n)$ indeed. Summarizing, Fig. 8 represents the FSE system for the cyclic-prefix based channel. This is a perfect equalizer reproducing $s(2 n)$ exactly in absence of channel noise.
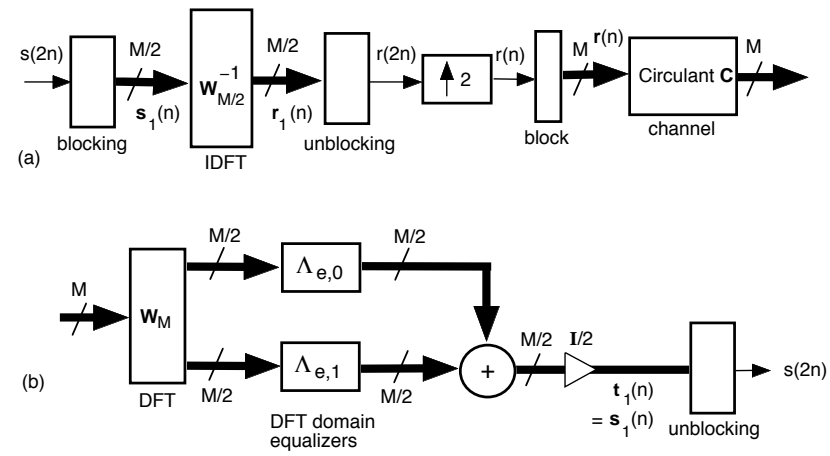

Figure 8. Rearrangement of the cyclic-prefix based FSE system. (a) Transmitter, and (b) receiver.

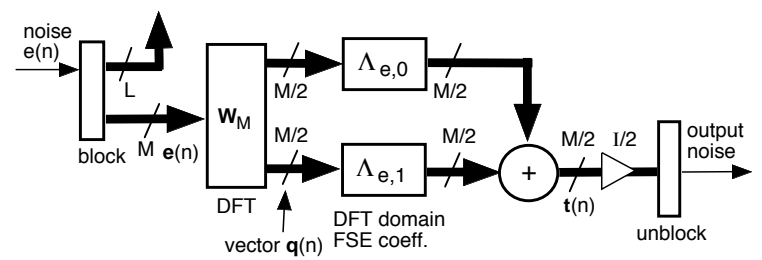

Figure 9. Processing of channel noise.

Noise reduction. The receiver is shown separately in Fig. 9 for noise analysis. The last $M$ components of the blocked version of $e(n)$ are collected into a vector $\mathbf{e}(n)$ which is transformed by the DFT matrix into the vector $\mathbf{q}(n)$. Assuming $e(n)$ is wide sense stationary, then so are $\mathbf{e}(n)$ and $\mathbf{q}(n)$, and the $M \times M$ autocorrelation matrix $\mathbf{R}_{\mathbf{q q}}$ of $\mathbf{q}(n)$ can be calculated. The components of $\mathbf{q}(n)$ are multiplied by the diagonal elements $E[k]$ of the matrices $\boldsymbol{\Lambda}_{e, 0}$ and $\boldsymbol{\Lambda}_{e, 1}$, and pairs of components separated by $M / 2$ are added to form the vector $\mathbf{t}(n)$. The output noise is the unblocked version of $0.5 \mathbf{t}(n)$. We have $t_{k}(n)=q_{k}(n) E[k]+q_{k+M / 2}(n) E[k+M / 2]$. For fixed $C[k]$ and $C[k+M / 2]$ we can optimize the coefficients $E[k]$ and $E[k+M / 2]$ subject to the constraint (3) such that $\mathcal{E}\left[\left|t_{k}(n)\right|^{2}\right]$ is minimized. Define the vectors $\mathbf{v}_{k}=$ $\left[\begin{array}{ll}E^{*}[k] & E^{*}[k+M / 2\end{array}\right]^{T}, \mathbf{q}_{k}=\left[\begin{array}{ll}q_{k}(n) & q_{k+M / 2}(n)\end{array}\right]^{T}$, and $\mathbf{c}_{k}=\left[\begin{array}{ll}C[k] & C[k+M / 2]\end{array}\right]^{T}$. Then $\mathcal{E}\left[\left|t_{k}(n)\right|^{2}\right]=$ $\mathbf{v}_{k}^{\dagger} \mathcal{E}\left[\mathbf{q}_{k} \mathbf{q}_{k}^{\dagger}\right] \mathbf{v}_{k}=\mathbf{v}_{k}^{\dagger} \mathbf{R}_{k} \mathbf{v}_{k}$. The constraint (3) can be written as $\mathbf{v}_{k}^{\dagger} \mathbf{c}_{k}=2$. Assuming $\mathbf{R}_{k}$ is nonsingular, we can show that $\mathcal{E}\left[\left|t_{k}(n)\right|^{2}\right]$ is minimized subject to this constraint if the equalizer vector is chosen as $\mathbf{v}_{k}=2 \mathbf{R}_{k}^{-1} \mathbf{c}_{k} / \mathbf{c}_{k}^{\dagger} \mathbf{R}_{k}^{-1} \mathbf{c}_{k}$.

\section{EXAMPLES AND CONCLUDING REMARKS}

Consider a channel of order $L=16$, and coefficients $c(n)$ given by $0.8860,0.1743,-0.5374,-0.1600,0.2175,0.0947$, $-0.0281,0.3735,0.7750,0.6480,0.5210,0.2151,-0.0908$, $-0.5296,-0.9683,-0.1643,0.6398$. The coefficients for the SSE case are obtained by retaining only the even coefficients, that is, $0.8860,-0.5374$, and so forth. We take $M=128$ and assume the channel noise is white. The scatter diagram for a 64-QAM constellation is shown in Fig. 10 for cyclic prefixed systems with SSE as well as noise-optimized FSE. The SNR at the channel output was fixed at $27 \mathrm{~dB}$ in both cases. The probabilities of error are $1.3 \times 10^{-2}$ and $4.9 \times 10^{-5}$ respectively. This clearly demonstrates the usefulness of the cyclic prefix FSE equalizer over the SSE system. It will be interesting to see how much further improvement can be obtained if the fractional sampling ratio is increased from two to a more general integer $K$.

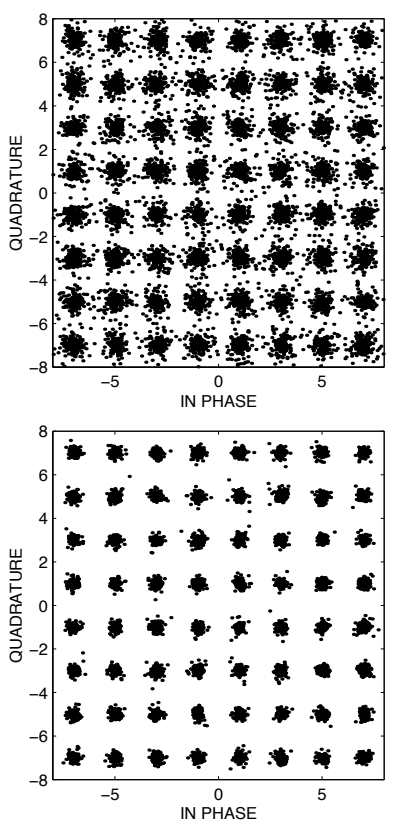

Figure 10. Results of equalization. Top: symbol spaced equalizer, and bottom: fractionally spaced equalizer.

\section{REFERENCES}

[1] Bingham, J. A. C. "Multicarrier modulation for data transmission: an idea whose time has come," IEEE Comm. Mag., pp. 5-14, May 1990.

[2] A. Papoulis, Signal analysis, McGraw Hill, 1977.

[3] Peled, A., and Ruiz, A. "Frequency domain data transmission using reduced computational complexity algorithms," IEEE ICASSP, pp. 964-967, Denver, CO, Apr. 1980.

[4] Treichler, J. R., Fijalkow, I., and Johnson, C. R., Jr., "Fractionally spaced equalizers: how long should they be?" IEEE SP Mag., pp. 65-81, May 1996.

[5] Starr, T., Cioffi, J. M., and Silverman, P. J. Understanding DSL technology, Prentice Hall, Inc., 1999.

[6] Vaidyanathan, P. P. Multirate systems and filter banks, Prentice Hall, Inc., 1993. 